\title{
ESTUDO BIOMÉTRICO DE RATOS ALIMENTADOS COM DOIS TIPOS DE DIETA
}

Alice Cristina Antonio dos Santos, Ana Carolina Tocilo Lopes,Gláucia de Cássia Xavier da Cruz, Bruna Corral Garcia, Fábio Yoshikazu Kodama, Regina Celi Trindade Camargo, José Carlos Silva Camargo Filho

Laboratório de Análise da Plasticidade Muscular (LAPMUS) do Departamento de Fisioterapia da Faculdade de Ciências e Tecnologia FCT/UNESP - Campus de Presidente Prudente. alice_fisioterapia@yahoo.com.br.

\section{RESUMO}

A dieta hipercalórica é tida como fator de risco para o desenvolvimento de doenças cardiovasculares, sendo preocupante para a saúde pública mundial. O objetivo do presente estudo foi comparar a biometria de ratos alimentados por dois tipos de dieta. Para tanto, foram utilizados 24 ratos Wistar machos com 90 dias com oferta de ração e água à vontade; divididos em G1 recebendo dieta normocalórica e G2, dieta hipercalórica. A ingesta alimentar foi mensurada diariamente. Após 18 semanas foram eutanasiados, com prévia mensuração de massa corpórea, comprimento naso-anal e cáculo do índice de Lee. Os dados foram tratados por estatística descritiva, com uso do teste de normalidade de Kolmogorov-Smirnov seguido por ANOVA - One Way e pós-teste de Tukey; com significância de 5\%. O grupo G2 apresentou maior ganho de peso e conseqüentemente índice de Lee superior ao G1, porém houve diminuição do consumo alimentar de G2 em comparação a G1.

Palavras-chave: ganho de peso, dieta hipercalórica, índice de Lee.

\section{BIOMETRIC STUDY OF RATS FED TWO DIETS}

\begin{abstract}
High caloric diet is regarded as a risk factor for developing cardiovascular diseases, is considered a global public health problem. The aim of this study was to compare the biometric parameters of rats fed with two diet types. Thus, 24 male Wistar rats (90 days) with intake of food and water ad libitum were divided into G1: receiving normocaloric diet, and G2: high caloric diet. Food intake was measured daily. After 18 weeks the rats were euthanized, and previous body mass, naso-anal length and Lee index were measured. Data were analyzed by descriptive statistics, followed by Kolmogorov-Smirnov normality test and ANOVA - One Way, using Tukey post hoc test, assuming $5 \%$ of significance. G2 had greater weight gain and therefore higher Lee index compared to $\mathrm{G} 1$; however, it was observed a decrease of food intake in $\mathrm{G} 2$ compared to $\mathrm{G} 1$.

Keywords: weight gain, high calorie diet, Lee index
\end{abstract}




\section{INTRODUÇÃO E JUSTIFICATIVA}

$O$ estilo de vida e as facilidades que $O$ mundo contemporâneo trouxeram influenciaram sobremaneira os hábitos alimentares, favorecendo uma dieta calórica com sobrecarga de carboidratos ou lipídios, conhecida como dieta "ocidentalizada" ou de "fast-food" (CESARETTI \& KOHLMANN JUNIOR, 2004; MENDONÇA \& ANJOS, 2004).

Em consequência desta nova tendência alimentar com alto consumo de gorduras, bem como o aumento da quantidade de colesterol total e lipoproteínas de alta densidade, ocorre acúmulo excessivo de gordura corporal, sobrepeso e obesidade, caracterizando ainda mais a dieta hipercalórica como um fator de risco para o desenvolvimento de doenças crônicas não transmissíveis (DCNT), como as doenças cardiovasculares e a Síndrome Metabólica (SM) (D'ORÁSIO \& MOURA, 2008; HARDMAN, 1999; MENDONÇA \& ANJOS, 2004; ZAMBON et al., 2009).

A quantidade de pessoas com sobrepeso e obesidade vem aumentando de forma significativa no Brasil e no mundo, por ser conseqüência de fatores endógenos (hereditariedade, fatores congênitos, psicogênicos, neurológicos e endócrinos) ou exógenos. Este último comporta a maioria dos casos, relacionados a fatores ambientais, principalmente a falta de atividade física e hábitos alimentares inadequados (ARAÚJO et al., 2009).

Por conseguinte, há aumento das taxas de mortalidade e interferência na qualidade de vida dos indivíduos (D'ORÁSIO \& MOURA, 2008; HARDMAN, 1999; MENDONÇA \& ANJOS, 2004; ZAMBON et al., 2009), tornando-se um assunto preocupante para a saúde pública mundial, pois pode aumentar a mortalidade geral em cerca de 1,5 vezes e a cardiovascular em cerca de 2,5 vezes (RIBEIRO FILHO et al., 2006; SBC, 2005).
Em roedores vêm sendo realizados estudos experimentais com promoção da obesidade por meio da ingesta de dieta hipercalórica a fim de reproduzir o comportamento nutricional humano e esclarecer esta abordagem alimentar (CESARETTI \& KOHLMANN JUNIOR, 2004; DINIZ et al., 2008; DUARTE et al., 2006; ESTADELLA et al., 2004; PRADA et al., 2004).

Neste sentido, o presente estudo visa comparar o efeito da administração prolongada de dieta normo e hipercalórica em ratos wistar.

\section{OBJETIVO}

Estudar e comparar a administração prolongada de dois tipos de dieta (normo e hipercalórica) em ratos Wistar, considerando o consumo alimentar, a massa corpórea e o índice de Lee.

\section{METODOLOGIA}

Foram utilizados 24 ratos machos da linhagem Wistar com 90 dias, provenientes do Biotério Central da UNESP, Campus de Botucatu, e mantidos no Biotério da FCT/UNESP (Campus de Presidente Prudente), dispostos em gaiolas plásticas coletivas de dimensão $30 \times 16$ x $19 \mathrm{~cm}$ contendo, no máximo, 5 animais.

Antes de serem utilizados, estes animais permaneceram no biotério por 15 dias para adaptação ao ambiente, com oferta de ração padrão e água da torneira à vontade, temperatura mantida em média de $22 \pm 2^{\circ} \mathrm{C}$, umidade relativa a $60 \%$ e ciclos claro-escuro de 12 horas, iniciando o período de claro às 07h00.

Após o período de adaptação ao Biotério, os animais foram pareados de acordo com o peso e divididos em dois grupos contendo 12 animais em cada. Durante todo o experimento os animais tiveram oferta de água de torneira e ração à vontade. Os animais do grupo G1 receberam dieta normocalórica, consistindo em 
ração comercial para ratos (Primor), contendo $23 \%$ de proteína, $49 \%$ de carboidrato, $4 \%$ de lipídeos, $5 \%$ de fibras, $7 \%$ de cinzas e $6 \%$ de vitaminas, totalizando $4,07 \mathrm{Kcal} / \mathrm{g}$; enquanto os animais do grupo $\mathrm{G} 2$ foram alimentados com dieta hipercalórica, que consistiu de uma mistura de alimentos hiperenergéticos, contendo $20 \%$ de proteína, $48 \%$ de carboidrato, $20 \%$ de lipídeos, $4 \%$ de fibras, $5 \%$ de vitaminas e minerais, totalizando $5,12 \mathrm{Kcal} / \mathrm{g}$. Para tanto, foi realizada a seguinte proporção: $15 \mathrm{~g}$ de ração padrão Primor (4,07 Kcal/g); $10 \mathrm{~g}$ de amendoim torrado (5,95 $\mathrm{Kcal} / \mathrm{g}) ; 10 \mathrm{~g}$ de chocolate ao leite $(5,4 \mathrm{Kcal} / \mathrm{g})$ e $5 \mathrm{~g}$ de biscoito de amido de milho $(4,25 \mathrm{Kcal} / \mathrm{g})$; triturados e misturados, formando pellets (ZAMBON et al., 2009).

Tabela 1. Composição das dietas ofertadas aos animais $(100 \mathrm{~g})$.

\begin{tabular}{ccc}
\hline Componentes & $\begin{array}{c}\text { Dieta } \\
\text { padrão }\end{array}$ & $\begin{array}{c}\text { Dieta } \\
\text { hipercalórica }\end{array}$ \\
\hline Proteína & $23 \%$ & $20 \%$ \\
Carboidrato & $49 \%$ & $48 \%$ \\
Lipídeos & $4 \%$ & $20 \%$ \\
Fibras & $5 \%$ & $4 \%$ \\
Cinzas & $7 \%$ & - \\
Vitaminas e & $6 \%$ & $5 \%$ \\
minerais & & \\
\hline
\end{tabular}

Durante o experimento, os animais foram pesados uma vez por semana e a ingesta alimentar foi mensurada diariamente. $\mathrm{O}$ consumo alimentar foi calculado por meio da diferença entre a ração ofertada e as sobras. Para o cálculo da evolução da massa corporal foi utilizada a seguinte fórmula: Delta $(\Delta \%)=[($ Massa final Massa inicial /Massa inicial) $X$ 100] (BERNARDES et al., 2004; ZAMBON et al., 2009).

Após 18 semanas de aplicação da dieta, os animais foram anestesiados, avaliados biometricamente com a mensuração da massa corpórea e do comprimento naso-anal (AGUILA et al, 2002). Posteriormente, estes dados foram utilizados para o cálculo do índice de Lee de cada animal, que é a razão da raiz cúbica da massa corporal $(\mathrm{g})$ pelo comprimento naso-anal $(\mathrm{cm})$ e multiplicando-se por 10, que equivale ao índice de massa corporal (IMC) obtido para humanos (ARAÚJO et al., 2009).

Os dados obtidos foram tratados por análise estatística descritiva, dispostas como média e desvio padrão. Foi aplicado o teste de normalidade de Kolmogorov-Smirnov seguido por análise de variância (ANOVA - One Way) e pósteste de Tukey; com nível de significância de 5\%.

\section{RESULTADOS}

A tabela 2 descreve os resultados de ganho de peso $(\mathrm{g})$, comprimento corporal $(\mathrm{cm})$ e índice de Lee.

O comprimento dos animais não apresentou diferença significante, porém os animais do grupo G2 apresentaram maior ganho de peso e conseqüentemente índice de Lee superior ao $\mathrm{G} 1$.

Os animais do grupo G2 apresentaram menores valores de consumo alimentar em comparação ao $\mathrm{G} 1$, apesar de terem apresentado maior ingesta no início do fornecimento da dieta.

Tabela 2. Ganho de peso, comprimento corporal, índice de Lee e ingesta alimentar.

\begin{tabular}{cccc}
\hline & G1 & G2 & p \\
\hline Gpeso (g) & $33,81 \pm 7,39$ & $58,78 \pm 13,29$ & $<0,0001$ \\
Comp (cm) & $27,15 \pm 0,67$ & $27,41 \pm 0,97$ & 0,4892 \\
iLee & $59,53 \pm 3,96$ & $69,84 \pm 5,77$ & 0,0001 \\
Cons (g) & $29,27 \pm 4,14$ & $22,72 \pm 3,77$ &
\end{tabular}

Ganho de peso (Gpeso), Comprimento naso-anal (comp), Índice de Lee (iLee), Média de consumo alimentar diário (Cons) 


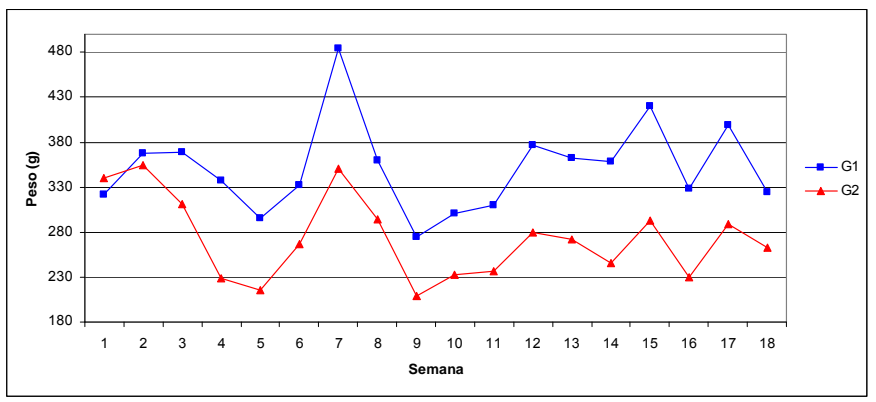

Figura 1. Ingesta alimentar dos animais.

\section{DISCUSSÃO}

Sabe-se que a obesidade tem apresentado valores crescentes na população mundial, provocando diversos problemas de saúde, incluindo riscos cardiovasculares, diabetes mellitus e outras doenças crônico-degenerativas, tanto em homens quanto em mulheres (ARAÚJO et al., 2009).

A maior parte das doenças decorrentes de obesidade está relacionada ao aumento de adiposidade visceral, sendo a gordura ingerida em excesso e a falta de atividade física as causas deste acúmulo (ZAMBON et al., 2009).

No presente estudo, optou-se pelo modelo de obesidade exógena, que mais se assemelha à obesidade humana, pois tem produzido aumento de peso corporal de $30-40 \%$ e de gordura visceral (CESARETTI \& KOHLMANN JUNIOR, 2004). Para tanto, administrou-se uma dieta hipercalórica aos animais substituindo a ração convencional por uma ração formulada, associando-se ração padrão a substâncias altamente calóricas, no caso chocolate, amendoim e biscoito.

A literatura apresenta grande divergência com relação à massa corpórea de ratos submetidos a dietas hipercalóricas. No presente estudo, notou-se que os animais do grupo G2 apresentaram maior índice de ganho de peso comparado ao $\mathrm{G} 1$, o que acorda com o estudo de Duarte et al. (2006).

Em humanos, tem-se o índice de massa corpórea que relaciona a disposição de tecido adiposo no organismo com obesidade, pois a distribuição da gordura corporal, em particular a gordura visceral (GV) desempenha um papel central na fisiopatologia da SM (RIBEIRO FILHO et al., 2006).

Semelhante ao que ocorre em humanos, as pesquisas com animais relacionam dietas hipercalóricas, obesidade e quantidades elevadas de tecido adiposo por meio do cálculo do índice de Lee (SOUZA et al., 2001). No presente estudo, o grupo G2 apresentou índice de Lee superior ao G1, caracterizando o animal como obeso devido à ingestão da dieta (DUARTE et al, 2006).

Pôde-se observar que com o passar do tempo o consumo alimentar dos animais diminuiu. Esta diminuição sugere aumento da saciedade, pois dietas ricas em gordura apresentam diminuição na eficiência alimentar e aumento da eficiência metabólica, devido aos altos níveis de substratos metabólicos plasmáticos, como glicose e triglicerídeos (BERNARDES et al, 2004; ZAMBON et al., 2009).

\section{CONCLUSÃO}

Em conclusão observamos que este modelo de dieta "ocidentalizada" foi capaz de provocar alterações significantes na biometria dos animais, além de a quantidade de alimento ingerido diminuir com o passar do tempo devido a sua alta concentração lipídica.

\section{REFERÊNCIAS}

Águila MB, Loureiro CC, Pinheiro AR, Mandarimde-Lacerda CA. Metabolismo lipídico de ratos alimentados com diferentes tipos de lipídios. Arq Bras Cardiol 2002, 78(1): 25-31.

Araújo GG, Araújo MB, DAngelo RA, Manchado FB, Mota CSA, Ribeiro C, Mello MAR. Máxima Fase estável de Lactato em ratos obesos de 
Ambos os gêneros. Rev Bras Med Esporte 2009, 15(1): 46-49.

Bernardes D, Manzoni MSJ, Souza CP, Tenório N, Damaso AR. Efeitos da dieta hiperlipídica e do treinamento de natação sobre o metabolismo de recuperação ao exercício em ratos. Rev Bras Educ Fís Esp 2004, 18(2): 191-200.

Cesaretti MLR, Kohlmann Junior O. Modelos Experimentais de Resistência à Insulina e Obesidade: Lições Aprendidas. Arq Bras Endocrinol Metab 2006; 50(2): 190-197.

Diniz YS, Burneiko RM, Seiva FRF, Almeida FQA, Galhardi CM, Novelli Filho JLVB, Mani F, Novelli ELB. Diet compounds, glycemic index and obesity-related cardiac effects. Intern J Cardiol 2008 , 124: 92-99.

http://dx.doi.org/10.1016/j.ijcard.2006.12.025

D’Orásio AC, Moura MJCS. Efeitos da obesidade induzida por dieta hipercalórica sobre a reatividade vascular na ausência do endotélio. Anais do XIII Encontro de Iniciação Científica da PUC-Campinas, 2008

Duarte ACGO, Fonseca DF, Manzoni MSJ, Soave CF, Sene-Fiorese M, Damaso AR, Cheik NC. Dieta hiperlipídica e capacidade secretória de insulina em ratos. Rev Nutr 2006, 19(3): 341-348.

Estadella D, Oyama LM, Damaso AR, Ribeiro EB, Nascimento CMO. Effect of Palatable Hyperlipidic Diet on Lipid Metabolism of Sedentary and Exercised Rats. Nutrition 2004, 20: 218-224. http://dx.doi.org/10.1016/j.nut.2003.10.008

Hardman AE. Physical activity, obesity and blood lipids. Int J Obes 1999, 23(Suppl 3): S64-S71. http://dx.doi.org/10.1038/sj.ijo.0800886

Mendonça CP, Anjos LA. Aspectos das práticas alimentares e da atividade física como determinantes do crescimento do sobrepeso/obesidade no Brasil. Cad. Saúde Pública [online] 2004; 20(3): 698-709.
Prada PO, Zecchin HG, Gasparetti AL, Torsoni MA, Ueno M, Hirata AE, Amaral MEC, Höer NF, Boschero AC, Saad MJA. Western diet modulates insulin signaling. Endocrinology 2005; 146(3):1576-1587.

http://dx.doi.org/10.1210/en.2004-0767

Ribeiro Filho FF, Mariosa LS, Ferreira SRGF, Zanella MT. Gordura Visceral e Síndrome Metabólica: mais que uma simples associação. Arq Bras Endocrinol Metab 2006, 50(2): 230-238.

Sociedade Brasileira de Cardiologia. I Diretriz Brasileira de Diagnóstico e Tratamento da Síndrome Metabólica. Arq Bras Cardiol 2005, 84(Supl I): 8-11. http://dx.doi.org/10.1590/S0004$\underline{27302006000200009}$

Souza F, Marchesini JB, Campos ACL, Malafaia O, Monteiro OG, Ribeiro FB, Alves HFP, Siroti JF, Meister $\mathrm{H}$, Mathias PCF. Efeito da vagotomiatroncular em ratos injetados na fase neonatal com glutamato monossódico: estudo biométrico. Acta Cir Bra 2001, 16(1): 32-45.

Zambon L, Duarte F O, Freitas LF, Scarmagnani FRR, Damaso A, Duarte ACGO, Sene-fiorese M. Efeitos de dois tipos de treinamento de natação sobre a adiposidade e o perfil lipídico de ratos obesos exógenos. Rev Nutr 2009, 22(5): 707-715. http://dx.doi.org/10.1590/S1415$\underline{52732009000500011}$ 